\title{
Asymptotic structure of viscous incompressible flow around a rotating body, with nonvanishing flow field at infinity.
}

\author{
Paul Deuring*, Stanislav Kračmar ${ }^{\dagger}$, Sárka Nečasová ${ }^{\ddagger}$
}

\begin{abstract}
We consider weak ("Leray") solutions to the stationary Navier-Stokes system with Oseen and rotational terms, in an exterior domain. It is shown the velocity may be split into a constant times the first column of the fundamental solution of the Oseen system, plus a remainder term decaying pointwise near infinity at a rate which is higher than the decay rate of the Oseen tensor. This result improves the theory by M. Kyed, Asymptotic profile of a linearized flow past a rotating body, Q. Appl. Math. 71 (2013), 489-500.
\end{abstract}

AMS subject classifications. 35Q30, 65N30, 76D05.

Key words. Stationary incompressible Navier-Stokes system, rotating body, pointwise decay, asymptotic profile.

\section{Introduction}

Let $\mathfrak{D} \subset \mathbb{R}^{3}$ be an open bounded set. Suppose this set describes a rigid body moving with constant nonzero translational and angular velocity in an incompressible viscous fluid. Then the flow aroung this body with respect to a frame attached to this body is governed by the following set of non-dimensional equations (see [24]),

$$
-\Delta u+\tau \partial_{1} u+\tau(u \cdot \nabla) u-(\omega \times x) \cdot \nabla u+\omega \times u+\nabla \pi=f, \operatorname{div} u=0,
$$

in the exterior domain $\overline{\mathfrak{D}}^{c}:=\mathbb{R}^{3} \backslash \overline{\mathfrak{D}}$, supplemented by a decay condition at infinity,

$$
u(x) \rightarrow 0 \text { for }|x| \rightarrow \infty,
$$

and suitable boundary conditions on $\partial \mathfrak{D}$. These latter conditions need not be specified here because they are not relevant in the context of the work at hand. In (1.1) and (1.2), the functions $u: \overline{\mathfrak{D}}^{c} \mapsto \mathbb{R}^{3}$ and $\pi: \overline{\mathfrak{D}}^{c} \mapsto \mathbb{R}$ are the unknown relative velocity and pressure field of the fluid, respectively, whereas the function $f: \overline{\mathfrak{D}}^{c} \mapsto \mathbb{R}^{3}$ stands for a prescribed volume force acting on the fluid. The vector $\tau(-1,0,0)$ represent the uniform velocity of

\footnotetext{
${ }^{*}$ Univ Lille Nord de France, 59000 Lille, France; ULCO, LMPA, 62228 Calais cédex, France.

${ }^{\dagger}$ Department of Technical Mathematics, Czech Technical University, Karlovo nám. 13, 12135 Prague 2, Czech Republic

${ }^{\ddagger}$ Mathematical Institute of the Academy of Sciences of the Czech Republic, Žitná 25, 11567 Praha 1, Czech Republic
} 
the flow at infinity or the velocity of the body, depending on the physical situation, and $\omega:=\varrho \cdot(1,0,0)$ corresponds to the constant angular velocity of the body. In particular the vectors of translational and angular velocity are parallel. From a physical point of view this assumption is natural for a steady flow. The parameters $\tau \in(0, \infty)$ and $\varrho \in \mathbb{R} \backslash\{0\}$ are dimensionless quantities that can be identified with the Reynolds and Taylor number, respectively. They will be considered as fixed, like the domain $\mathfrak{D}$.

We are interested in "Leray solutions" of (1.1), (1.2), that is, weak solutions characterized by the conditions $u \in L^{6}\left(\overline{\mathfrak{D}}^{c}\right)^{3} \cap W_{\text {loc }}^{1,1}\left(\overline{\mathfrak{D}}^{c}\right)^{3}, \nabla u \in L^{2}\left(\overline{\mathfrak{D}}^{c}\right)^{9}$ and $\pi \in L_{l o c}^{2}\left(\overline{\mathfrak{D}}^{c}\right)$. The relation $u \in L^{6}\left(\overline{\mathfrak{D}}^{c}\right)^{3}$ means that (1.2) is verified in a weak way. Such solutions exist for data of arbitrary size if some smoothness of the boundary of $\mathfrak{D}$ is required, and if suitable regularity conditions are imposed on $f$ and on the data on the boundary ([26, Theorem IX.3.1]). It is well known by now ([27], [7]) that the velocity part $u$ of a Leray solution $(u, \pi)$ to (1.1), (1.2) decays for $|x| \rightarrow \infty$ as expressed by the estimates

$$
|u(x)| \leq C(|x| s(x))^{-1}, \quad|\nabla u(x)| \leq C(|x| s(x))^{-3 / 2}
$$

for $x \in \mathbb{R}^{3}$ with $|x|$ sufficiently large, where $s(x):=1+|x|-x_{1}\left(x \in \mathbb{R}^{3}\right)$ and $C>$ 0 a constant independent of $x$. The factor $s(x)$ may be considered as a mathematical manifestation of the wake extending downstream behind a body moving in a viscous fluid. In view of (1.3), it is natural to ask how an asymptotic expansion of $u$ for $|x| \rightarrow \infty$ might look like. As far as we know, up to now there are two answers to this question. The first is due to Kyed [49, who showed that

$$
u_{j}(x)=\gamma E_{j 1}(x)+R_{j}(x), \quad \partial_{l} u_{j}(x)=\gamma \partial_{l} E_{j 1}(x)+S_{j l}(x) \quad\left(x \in \overline{\mathfrak{D}}^{c}, 1 \leq j, l \leq 3\right),
$$

where $E: \mathbb{R}^{3} \backslash\{0\} \mapsto \mathbb{R}^{4} \times \mathbb{R}^{3}$ denotes a fundamental solution to the Oseen system

$$
-\Delta v+\tau \partial_{1} v+\nabla \varrho=f, \operatorname{div} v=0 \quad \text { in } \mathbb{R}^{3} .
$$

The definition of the function $E$ is stated in Section 2. As becomes apparent from this definition, the term $E_{j 1}(x)$ may be expressed explicitly in terms of elementary functions. The coefficient $\gamma$ is also given explicitly, its definition involving the Cauchy stress tensor. The remainder terms $R$ and $S$ are characterized by the relations $R \in L^{q}\left(\overline{\mathfrak{D}}^{c}\right)^{3}$ for $q \in$ $(4 / 3, \infty), S \in L^{q}\left(\overline{\mathfrak{D}}^{c}\right)^{3}$ for $q \in(1, \infty)$. Since it is known from [23, Section VII.3] that $E_{j 1} \mid B_{r}^{c} \notin L^{q}\left(B_{r}^{c}\right)$ for $r>0, q \in[1,2]$, and $\partial_{l} E_{j 1} \mid B_{r}^{c} \notin L^{q}\left(B_{r}^{c}\right)$ for $r>0, q \in[1,4 / 3], j, l \in$ $\{1,2,3\}$, the function $R$ decays faster than $E_{j 1}$, and $S_{j l}$ faster than $\partial_{l} E_{j 1}$, in the sense of $L^{q}$-integrability. Thus the equations in (1.4) may in fact be considered as asymptotic expansions of $u$ and $\nabla u$, respectively. The theory in [49] is valid under the assumption that $u$ verifies the boundary conditions

$$
u(x)=e_{1}+(\omega \times x) \text { for } x \in \partial \mathfrak{D} .
$$

Reference [49] does not deal with pointwise decay of $R$ and $S$, nor does it indicate whether $S=\nabla R$. The second answer to the question of how $u$ may be expanded asymptotically for $|x| \rightarrow \infty$ is given in reference [9], which states that for $x \in \overline{B_{S_{1}}}, 1 \leq j \leq 3$,

$$
u_{j}(x)=\sum_{k=1}^{3} \beta_{k} Z_{j k}(x, 0)+\left(\int_{\partial \Omega} u \cdot n d o_{x}\right) x_{j}\left(4 \pi|x|^{3}\right)^{-1}+\mathfrak{F}_{j}(x) .
$$


Here $S_{1}$ is a sufficiently large positive real number, $\left(Z_{j k}\right)_{1 \leq j, k \leq 3}$ is the tensor velocity part of the fundamental solution constructed by Guenther, Thomann [57] for the linearization

$$
-\Delta v+\tau \partial_{1} v-(\omega \times x) \cdot \nabla v+\omega \times v+\nabla \varrho=f, \operatorname{div} v=0
$$

of (1.1) (see Section 2 for the definition of $Z$ ), and $\mathfrak{F}$ is a function from $C^{1}\left({\overline{B_{S_{1}}}}^{c}\right)^{3}$ given explicitly in terms of $Z, u$ and $\pi$ (Theorem 3.1). As is shown in [9], this function $\mathfrak{F}$ decays pointwise, in the sense that

$$
\lim _{|x| \rightarrow \infty}\left|\partial^{\alpha} \mathfrak{F}(x)\right|=O\left((|x| s(x))^{-3 / 2-|\alpha| / 2} \ln (2+|x|)\right) \quad \text { for } \alpha \in \mathbb{N}_{0}^{3} \text { with }|\alpha| \leq 1 .
$$

It is known from [4, Theorem 2.19] - and restated below in Corollary 2.3 - that

$$
\lim _{|x| \rightarrow \infty}\left|\partial^{\alpha} Z(x, 0)\right|=O\left((|x| s(x))^{-1-|\alpha| / 2}\right) \quad\left(\alpha \in \mathbb{N}_{0}^{3} \text { with }|\alpha| \leq 1\right) .
$$

So, if the decay rate in (1.9) is sharp, equation (1.7) may be considered as an asymptotic expansion in the usual sense: the remainder exhibits a faster pointwise decay than the leading term. The coefficients $\beta_{1}, \beta_{2}, \beta_{3}$ in (1.7) are given explicitly in terms of $u, \pi$ and $f$. The theory in [9] does not impose any boundary condition on $u$ or $\pi$. However, since the definition of the term $Z(x, 0)$ involves an integral over $(0, \infty)$, the leading term $\sum_{k=1}^{3} \beta_{k} Z_{j k}(x, 0)$ in (1.7) is not as explicit as one would like it to be. More details on the theory from [9] may be found in Theorem 3.1 below, where the main result from [9] is restated.

In the work at hand, we show that $Z_{j 1}(x, 0)=E_{j 1}(x)$ for $x \in \mathbb{R}^{3} \backslash\{0\}, 1 \leq j \leq 3$, and $\lim _{|x| \rightarrow \infty}\left|\partial_{x}^{\alpha} Z_{j k}(x, 0)\right|=O\left((|x| s(x))^{-3 / 2-|\alpha| / 2}\right)$ for $1 \leq j \leq 3, k \in\{2,3\}$ (Corollary 4.5, Theorem 5.1). Thus, setting

$$
\mathfrak{G}_{j}(x):=\sum_{k=2}^{3} \beta_{k} Z_{j k}(x, 0)+\mathfrak{F}_{j}(x) \quad\left(x \in{\overline{B_{S_{1}}}}^{c}, 1 \leq j \leq 3\right),
$$

we may deduce from (1.7) that

$u_{j}(x)=\beta_{1} E_{j 1}(x)+\left(\int_{\partial \Omega} u \cdot n d o_{x}\right) x_{j}\left(4 \pi|x|^{3}\right)^{-1}+\mathfrak{G}_{j}(x) \quad\left(x \in{\overline{B_{S_{1}}}}^{c}, 1 \leq j \leq 3\right)$

and

$$
\lim _{|x| \rightarrow \infty}\left|\partial^{\alpha} \mathfrak{G}(x)\right|=O\left((|x| s(x))^{-3 / 2-|\alpha| / 2} \ln (2+|x|)\right) \quad \text { for } \quad \alpha \in \mathbb{N}_{0}^{3} \text { with }|\alpha| \leq 1
$$

(Theorem 3.2, Corollary 3.1). If we compare how the coefficient $\gamma$ from (1.4) is defined in [49, and the coefficient $\beta_{1}$ from (1.11) in [9] (see Theorem 3.1 below), and if we take account of the boundary condition (1.6) satisfied by $u$ in [49], we see that $\gamma$ and $\beta_{1}$ coincide. Thus the relations in (1.11) and (1.12) provide a synthesis of the theories in [49] and [9]: the leading terms in (1.4) and (1.11) are identical, and the remainder in (1.11) decays pointwise for $|x| \rightarrow \infty$, its rate of decay being $|x|^{-2-|\alpha|}+(|x| s(x))^{-3 / 2-|\alpha| / 2} \ln (2+|x|)$. It is shown in 44 - and restated below in Theorem 2.3 - that

$$
\lim _{|x| \rightarrow \infty} \partial^{\alpha} E_{j 1}(x)=O\left((|x| s(x))^{-1-|\alpha| / 2}\right) \quad \text { for } \alpha \in \mathbb{N}_{0}^{3} \text { with }|\alpha| \leq 1
$$


(44, (1.14)]). The theory in [44] additionally yields that the decay rate $O(|x| s(x))^{-1-|\alpha| / 2}$ in (1.13) is sharp. Therefore it follows from (1.12) that equation (1.11) is in fact an asymptotic expansion of $u_{j}(x)$ for $|x| \rightarrow \infty$, with the remainder vanishing faster for large values of $|x|$ than the leading term $\beta_{1} E_{j 1}(x)$. It further follows that the decay rates of $u$ and $\nabla u$ given by (1.3) are sharp, too. The reader may wish to check on the basis of the theory in 44] whether some part of $E_{j 1}(x)$ may be split off and put into the remainder term. (This is possible for $\nabla E_{j 1}(x)$ but not for $E_{j 1}(x)$ if a decay rate as in (1.12) is to be maintained.)

We further remark that in the case of a rigid body which only rotates but does not translate, more detailed asymptotic expansions are available ([16] - [18]). Any reader interested in further results on the asymptotic behaviour of viscous incompressible flow around rotating bodies is referred to [3]- 6], [8], [12] - 15], 19] - 22], 25], 26], 28][43], 45] - 48], [51] - [53], [57].

\section{Notation. Definition of fundamental solutions. Auxiliary results.}

By || we denote the Euclidean norm in $\mathbb{R}^{3}$ and the length $\alpha_{1}+\alpha_{2}+\alpha_{3}$ of a multiindex $\alpha \in \mathbb{N}_{0}^{3}$. Put $e_{1}:=(1,0,0)$. For $r>0$, we set $B_{r}:=\left\{y \in \mathbb{R}^{3}:|y|<r\right\}$. If $A \subset \mathbb{R}^{3}$, we put $A^{c}:=\mathbb{R}^{3} \backslash A$. Recall the abbreviation $s(x):=1+|x|-x_{1}\left(x \in \mathbb{R}^{3}\right)$ introduced in Section 1 .

If $A \subset \mathbb{R}^{3}$ is open, $p \in[1, \infty)$ and $k \in \mathbb{N}$, we write $W^{k, p}(A)$ for the usual Sobolev space of order $k$ and exponent $p$. If $B \subset \mathbb{R}^{3}$ is again an open set, we define $L_{l o c}^{p}(B), W_{l o c}^{k, p}(B)$ as the set of all functions $v: B \mapsto \mathbb{R}$ such that $\left.v\right|_{U} \in L^{p}(U)$ and $\left.v\right|_{U} \in W^{k, p}(U)$, respectively, for any open bounded set $U \subset \mathbb{R}^{3}$ with $\bar{U} \subset B$. We write $\mathfrak{S}\left(\mathbb{R}^{3}\right)$ for the usual space of rapidly decreasing functions in $\mathbb{R}^{3}$; see [50, p. 138] for example. For the Fourier transform $\widehat{g}$ of a function $g \in L^{1}\left(\mathbb{R}^{3}\right)$, we choose the definition $\widehat{g}(\xi):=(2 \pi)^{-3 / 2} \int_{\mathbb{R}^{3}} e^{-i \xi x} g(x) d x\left(\xi \in \mathbb{R}^{3}\right)$. This fixes the definition of the Fourier transform of a tempered distribution as well.

The numbers $\tau \in(0, \infty), \varrho \in \mathbb{R} \backslash\{0\}$ introduced in Section 1 will be kept fixed throughout. Recall that the vector $\omega$ is given by $\omega:=r \cdot e_{1}$. We introduce a matrix $\Omega \in \mathbb{R}^{3 \times 3}$ by setting

$$
\Omega:=\varrho\left(\begin{array}{rrr}
0 & 0 & 0 \\
0 & 0 & -1 \\
0 & 1 & 0
\end{array}\right) .
$$

Note that $\omega \times x=\Omega \cdot x$ for $x \in \mathbb{R}^{3}$. We write $\mathfrak{C}$ for positive constants that may depend on $\tau$ or $\varrho$. Constants additionally depending on parameters $\sigma_{1}, \ldots, \sigma_{n} \in(0, \infty)$ for some $n \in \mathbb{N}$ are denoted by $\mathfrak{C}\left(\sigma_{1}, \ldots, \sigma_{n}\right)$. We state some inequalities involving $s(x)$ or $x-\tau t e_{1}$.

Lemma 2.1 ([2, Lemma 4.8]) $s(x-y)^{-1} \leq \mathfrak{C}(1+|y|) s(x)^{-1}$ for $x, y \in \mathbb{R}^{3}$.

Lemma 2.2 ([1, Lemma 2]) For $x \in \mathbb{R}^{3}, t \in(0, \infty)$, we have

$$
\left|x-\tau t e_{1}\right|^{2}+t \geq \mathfrak{C}\left[\chi_{[0,1]}(|x|)\left(|x|^{2}+t\right)+\chi_{(1, \infty)}(|x|)(|x| s(x)+t)\right] .
$$


Theorem 2.1 ([4, Theorem 2.19] ) Let $R_{1}, R_{2} \in(0, \infty)$ with $R_{1}<R_{2}, \nu \in(1, \infty)$. Then for $y \in B_{R_{2}}^{c}, z \in B_{R_{1}}$,

$$
\int_{0}^{\infty}\left(\left|y-\tau t e_{1}-e^{-t \Omega} \cdot z\right|^{2}+t\right)^{-\nu} d t \leq \mathfrak{C}\left(R_{1}, R_{2}, \nu\right)(|y| s(y))^{-\nu+1 / 2} .
$$

Theorem 2.2 Let $R \in(0, \infty)$. Then for $k \in\{0,1\}, x, y \in B_{R}$ with $x \neq y$,

$$
\int_{0}^{\infty}\left(\left|x-\tau t e_{1}-e^{-t \cdot \Omega} \cdot y\right|^{2}+t\right)^{-3 / 2-k / 2} d t \leq C(R)|x-y|^{-1-k} .
$$

Proof: See the last part of the proof of [3, Theorem 3.1]. Note that in [3, (3.7)] it should read $y+t U-e^{-t \Omega} \cdot z$ instead of $x$.

The next lemma is well known. It was already used in [18], for example. For the convenience of the reader, we give a proof.

Lemma 2.3 Let $t \in \mathbb{R}$. Then $e^{t \Omega}=\left(\begin{array}{rrr}1 & 0 & 0 \\ 0 & \cos (t \varrho) & -\sin (t \varrho) \\ 0 & \sin (t \varrho) & \cos (t \varrho)\end{array}\right)$.

Proof: Put $\widetilde{I}:=\left(\begin{array}{lll}0 & 0 & 0 \\ 0 & 1 & 0 \\ 0 & 0 & 1\end{array}\right), I:=\left(\begin{array}{lll}1 & 0 & 0 \\ 0 & 1 & 0 \\ 0 & 0 & 1\end{array}\right), A:=\left(\begin{array}{rrr}0 & 0 & 0 \\ 0 & 0 & -1 \\ 0 & 1 & 0\end{array}\right)$. It is easy to check that $\Omega=\varrho A, A^{2}=-\widetilde{I}, A^{3}=-A$ and $A^{4}=\widetilde{I}$. Therefore

$$
\begin{aligned}
& e^{t \Omega}=\sum_{i=0}^{\infty}(\varrho t A)^{i} / i !=I+\left(\sum_{i=1}^{\infty}(\varrho t)^{4 i} /(4 i) !\right) \widetilde{I}+\left(\sum_{i=0}^{\infty}(\varrho t)^{4 i+1} /(4 i+1) !\right) A \\
& \quad+\left(\sum_{i=0}^{\infty}(\varrho t)^{4 i+2} /(4 i+2) !\right)(-\widetilde{I})+\left(\sum_{i=0}^{\infty}(\varrho t)^{4 i+3} /(4 i+3) !\right)(-A) \\
& =I+\left(\sum_{j=1}^{\infty}(-1)^{j}(\varrho t)^{2 j} /(2 j) !\right) \widetilde{I}+\left(\sum_{j=0}^{\infty}(-1)^{j}(\varrho t)^{2 j+1} /(2 j+1) !\right) A \\
& =I+(\cos (\varrho t)-1) \widetilde{I}+\sin (\varrho t) A .
\end{aligned}
$$

This implies the lemma.

Next we introduce some fundamental solutions. Put

$$
N(x):=(4 \pi|x|)^{-1} \text { for } x \in \mathbb{R}^{3} \backslash\{0\}
$$

("Newton potential", fundamental solution of the Poisson equation in $\mathbb{R}^{3}$ ),

$$
\mathfrak{O}(x):=(4 \pi|x|)^{-1} e^{-\tau\left(|x|-x_{1}\right) / 2} \quad \text { for } x \in \mathbb{R}^{3} \backslash\{0\}
$$

(fundamental solution of the scalar Oseen equation $-\Delta v+\tau \partial_{1} v=g$ in $\mathbb{R}^{3}$ ),

$$
\mathfrak{O}^{(\lambda)}(x):=(4 \pi|x|)^{-1} e^{-\sqrt{\lambda+\tau^{2} / 4}|x|+\tau x_{1} / 2} \quad \text { for } \quad x \in \mathbb{R}^{3} \backslash\{0\}, \lambda \in(0, \infty)
$$


(fundamental solution of the scalar Oseen resolvent equation $-\Delta v+\tau \partial_{1} v+\lambda v=g$ in $\left.\mathbb{R}^{3}\right)$,

$$
K(x, t):=(4 \pi t)^{-3 / 2} e^{-|x|^{2} /(4 t)} \quad \text { for } x \in \mathbb{R}^{3}, t \in(0, \infty)
$$

(fundamental solution of the heat equation in $\mathbb{R}^{3}$ ),

$$
\begin{aligned}
& \psi(r):=\int_{0}^{r}\left(1-e^{-t}\right) t^{-1} d t \quad(r \in \mathbb{R}), \quad \Phi(x):=(4 \pi \tau)^{-1} \psi\left(\tau\left(|x|-x_{1}\right) / 2\right) \quad\left(x \in \mathbb{R}^{3}\right), \\
& E_{j k}(x):=\left(\delta_{j k} \Delta-\partial_{j} \partial_{k}\right) \Phi(x), \quad E_{4 k}(x):=x_{k}\left(4 \pi|x|^{3}\right)^{-1} \quad\left(x \in \mathbb{R}^{3} \backslash\{0\}, 1 \leq j, k \leq 3\right)
\end{aligned}
$$

(fundamental solution of the Oseen system (1.5), with $\left(E_{j k}\right)_{1 \leq j, k \leq 3}$ the velocity part and $\left(E_{4 k}\right)_{1 \leq k \leq 3}$ the pressure part). We further define

$$
F^{(\lambda)}(\xi):=(2 \pi)^{-3 / 2}\left(\lambda+|\xi|^{2}+i \tau \xi_{1}\right)^{-1} \text { for } \xi \in \mathbb{R}^{3}, \lambda \in(0, \infty)
$$

(Fourier transform of $\mathfrak{O}^{(\lambda)}$; see Theorem 4.1).

We recall some basic properties of these functions, beginning with a classical result.

Lemma 2.4 Let $f \in \mathfrak{S}\left(\mathbb{R}^{3}\right)$ and put $F(x):=\int_{\mathbb{R}^{3}} N(x-y) f(y) d y$ for $x \in \mathbb{R}^{3}$. Then $F \in C^{\infty}\left(\mathbb{R}^{3}\right)$ and $\partial^{\alpha} F(x)=\int_{\mathbb{R}^{3}} N(x-y) \partial^{\alpha} f(y) d y$ for $x \in \mathbb{R}^{3}, \alpha \in \mathbb{N}_{0}^{3}$.

Lemma 2.5 ([11]) $K \in C^{\infty}\left(\mathbb{R}^{3} \times(0, \infty)\right)$ and

$$
\left|\partial_{t}^{l} \partial_{x}^{\alpha} K(x, t)\right| \leq \mathfrak{C}(\alpha, l)\left(|x|^{2}+t\right)^{-3 / 2-|\alpha| / 2-l} e^{-|x|^{2} /(8 t)}
$$

for $x \in \mathbb{R}^{3}, t \in(0, \infty), \alpha \in \mathbb{N}_{0}^{3}, l \in \mathbb{N}_{0}$. In particular $K(\cdot, t) \in L^{1}\left(\mathbb{R}^{3}\right) \cap \mathfrak{S}\left(\mathbb{R}^{3}\right)$ for $t>0$.

Theorem $2.3([44]) E_{j k} \in C^{\infty}\left(\mathbb{R}^{3} \backslash\{0\}\right)$ and

$$
\left|\partial^{\alpha} E_{j k}(x)\right| \leq \mathfrak{C}(|x| s(x))^{-1-|\alpha| / 2} \max \left\{1,|x|^{-|\alpha| / 2}\right\}
$$

for $x \in \mathbb{R}^{3} \backslash\{0\}, 1 \leq j, k \leq 3, \alpha \in \mathbb{N}_{0}^{3}$ with $|\alpha| \leq 1$.

As a consequence of Theorem [2.3, we have $E_{j k} \in L_{l o c}^{1}\left(\mathbb{R}^{3} \backslash\{0\}\right)$ and $E_{j k} \mid B_{1}^{c}$ bounded $(1 \leq j, k \leq 3)$. The same properties are obvious for $N, \mathfrak{O}$ and $\mathfrak{O}^{(\lambda)}$. Moreover $|\Phi(x)| \leq$ $\mathfrak{C}(1+|x|) \quad\left(x \in \mathbb{R}^{3}\right)$. In view of these observations, the Fourier transforms of these functions will be considered as tempered distributions (which, of course, will turn out to be represented by functions). Following Solonnikov [55, (40)], we use Lemma 2.4 and 2.5 to introduce the velocity part $\left(T_{j k}\right)_{1 \leq j, k \leq 3}$ of a fundamental solution of the time-dependent Stokes system, setting

$$
T_{j k}(x, t):=\delta_{j k} K(x, t)+\partial_{j} \partial_{k}\left(\int_{\mathbb{R}^{3}} N(x-y) K(y, t) d y\right) \quad\left(x \in \mathbb{R}^{3}, t>0,1 \leq j, k \leq 3\right) .
$$

Lemma 2.6 ([55, Lemma 13], [54]) $T_{j k} \in C^{\infty}\left(\mathbb{R}^{3} \times(0, \infty)\right)$ and

$$
\left|\partial_{t}^{l} \partial_{x}^{\alpha} T_{j k}(x, t)\right| \leq \mathfrak{C}(\alpha, l)\left(|x|^{2}+t\right)^{-3 / 2-|\alpha| / 2-l}
$$

for $x \in \mathbb{R}^{3}, t \in(0, \infty), 1 \leq j, k \leq 3, \alpha \in \mathbb{N}_{0}^{3}, l \in \mathbb{N}_{0}$. 
Lemma 2.6 yields that $T_{j k}(\cdot, t) \in L^{2}\left(\mathbb{R}^{3}\right)$, but does not imply $T_{j k}(\cdot, t) \in L^{1}\left(\mathbb{R}^{3}\right)(t>0)$. So the Fourier transform of this function should be understood either as a transform of an $L^{2}$-function or as a tempered distribution. For us it will be convenient to use the second possibility. Put

$$
\Gamma_{j k}(x, y, t):=T\left(x-\tau t e_{1}-e^{-t \Omega} \cdot y, t\right) \cdot e^{-t \Omega} \quad \text { for } x, y \in \mathbb{R}^{3}, t>0 .
$$

The matrix-valued function $\left(\Gamma_{j k}\right)_{1 \leq j, k \leq 3}$ is the velocity part of a fundamental solution to the time-dependent variant of the linearization (1.8) of (1.1). This fundamental solution was constructed by Guenther, Thomann [57] via a procedure involving Kummer functions, an approach also used in [3] - 9]. However, Guenther, Thomann [57, (3.9)] showed that $\Gamma$ is given by (2.1) as well, thus providing an access to this function which is more convenient in many respects. For example, from Lemma 2.6 and (2.1) we immediately obtain

Corollary 2.1 Let $j, k \in\{1,2,3\}$. Then $\Gamma_{j k} \in C^{\infty}\left(\mathbb{R}^{3} \times \mathbb{R}^{3} \times(0, \infty)\right)$ and

$$
\left|\partial_{x}^{\alpha} \Gamma_{j k}(x, y, t)\right| \leq \mathfrak{C}(\alpha)\left(\left|x-\tau t e_{1}-e^{-t \Omega} \cdot y\right|^{2}+t\right)^{-3 / 2-|\alpha| / 2}
$$

for $x, y \in \mathbb{R}^{3}, t \in(0, \infty), \alpha \in \mathbb{N}_{0}^{3}$.

By Theorem 2.2 and Corollary 2.1, we have $\int_{0}^{\infty}|\Gamma(x, y, t)| d t<\infty$ for $x, y \in \mathbb{R}^{3}$ with $x \neq y$, so we may define

$$
Z_{j k}(x, y):=\int_{0}^{\infty} \Gamma(x, y, t) d t \quad \text { for } x, y \in \mathbb{R}^{3} \text { with } x \neq y, 1 \leq j, k \leq 3 .
$$

This function $Z$ was introduced on [57, p. 96] as the velocity part of a fundamental solution to (1.8). We collect the properties of $Z$ that will be needed in what follows.

Lemma 2.7 ([4, Lemma 2.15]) $Z \in C^{1}\left(\left(\mathbb{R}^{3} \times \mathbb{R}^{3}\right) \backslash \operatorname{diag}\left(\mathbb{R}^{3} \times \mathbb{R}^{3}\right)\right)^{3 \times 3}, \quad \partial x_{l} Z(x, y)=$ $\int_{0}^{\infty} \partial x_{l} \Gamma(x, y, t) d t$ for $x, y \in \mathbb{R}^{3}$ with $x \neq y, 1 \leq l \leq 3$.

Note that due to Theorem 2.2 and Corollary 2.1, we have $\int_{0}^{\infty}\left|\partial x_{l} \Gamma(x, y, t)\right| d t<\infty$ for $x, y, l$ as in Lemma 2.7.

Corollary 2.2 Let $R_{1}, R_{2} \in(0, \infty)$ with $R_{1}<R_{2}$. Then

$$
\left|\partial_{x}^{\alpha} Z(x, y)\right| \leq \mathfrak{C}\left(R_{1}, R_{2}\right)(|x| s(x))^{-1-|\alpha| / 2} \text { for } x \in B_{R_{2}}^{c}, y \in B_{R_{1}}, \alpha \in \mathbb{N}_{0}^{3} \text { with }|\alpha| \leq 1
$$

Proof: Lemma 2.7, Corollary 2.1, Theorem 2.1.

Corollary 2.3 The function $Z(\cdot, 0)$ belongs to $C^{1}\left(\mathbb{R}^{3} \backslash\{0\}\right)^{3 \times 3}$.

Let $S \in(0, \infty)$. Then $\left|\partial_{x}^{\alpha} Z(x, 0)\right| \leq \mathfrak{C}(S)(|x| s(x))^{-1-|\alpha| / 2}$ for $x \in B_{S}^{c}, \alpha \in \mathbb{N}_{0}^{3}$ with $|\alpha| \leq 1$.

Moreover $|Z(x, 0)| \leq \mathfrak{C}|x|^{-1}$ for $x \in B_{1} \backslash\{0\}$.

Proof: The first part of the corollary follows from Lemma 2.7 and Corollary 2.2. The last estimate is a consequence of Corollary 2.1 and Theorem 2.2.

Corollary 2.3 justifies to introduce the Fourier transform of $Z(\cdot, 0)$ in the sense of a tempered distribution. 


\section{Statement of our main result.}

It will be convenient to first recall the main result from [9].

Theorem 3.1 ([9, Theorem 3.1]) Let $\mathfrak{D} \subset \mathbb{R}^{3}$ be open, $p \in(1, \infty), f \in L^{p}\left(\mathbb{R}^{3}\right)^{3}$ with $\operatorname{supp}(f)$ compact. Let $S_{1} \in(0, \infty)$ with $\overline{\mathfrak{D}} \cup \operatorname{supp}(f) \subset B_{S_{1}}$.

Let $u \in L^{6}\left(\overline{\mathfrak{D}}^{c}\right)^{3} \cap W_{l o c}^{1,1}\left(\overline{\mathfrak{D}}^{c}\right)^{3}, \pi \in L_{\text {loc }}^{2}\left(\overline{\mathfrak{D}}^{c}\right)$ with $\nabla u \in L^{2}\left(\overline{\mathfrak{D}}^{c}\right)^{9}$, divu $=0$ and

$$
\begin{aligned}
& \int_{\overline{\mathfrak{D}}^{c}}\left[\nabla u \cdot \nabla \varphi+\left(\tau \partial_{1} u+\tau(u \cdot \nabla) u-(\omega \times z) \cdot \nabla u+\omega \times u\right) \cdot \varphi-\pi \operatorname{div} \varphi\right] d z \\
& =\int_{\overline{\mathfrak{D}}^{c}} f \cdot \varphi d z \quad \text { for } \varphi \in C_{0}^{\infty}\left(\overline{\mathfrak{D}}^{c}\right)^{3} .
\end{aligned}
$$

(This means the pair $(u, \pi)$ is a Leray solution to (1.1), (1.2).) Suppose in addition that

$$
\mathfrak{D} \text { is } C^{2} \text {-bounded, } \quad u\left|\partial \mathfrak{D} \in W^{2-1 / p, p}(\partial \mathfrak{D})^{3}, \quad \pi\right| B_{S_{1}} \backslash \overline{\mathfrak{D}} \in L^{p}\left(B_{S_{1}} \backslash \overline{\mathfrak{D}}\right) .
$$

Let $n$ denote the outward unit normal to $\mathfrak{D}$, and define

$$
\begin{aligned}
\beta_{k} & :=\int_{\overline{\mathfrak{D}}^{c}} f_{k}(y) d y \\
& +\int_{\partial \mathfrak{D}} \sum_{l=1}^{3}\left(-\partial_{l} u_{k}(y)+\delta_{k l} \pi(y)+\left(\tau e_{1}-\omega \times y\right)_{l} u_{k}(y)-\tau\left(u_{l} u_{k}\right)(y)\right) n_{l}(y) d o_{y}
\end{aligned}
$$

for $1 \leq k \leq 3$,

$$
\begin{aligned}
& \mathfrak{F}_{j}(x):=\int_{\overline{\mathfrak{D}}^{c}}\left[\sum_{k=1}^{3}\left(Z_{j k}(x, y)-Z_{j k}(x, 0)\right) f_{k}(y)-\tau \cdot \sum_{k, l=1}^{3} Z_{j k}(x, y)\left(u_{l} \partial_{l} u_{k}\right)(y)\right] d y \\
& +\int_{\partial \mathfrak{D}} \sum_{k=1}^{3}\left[\left(Z_{j k}(x, y)-Z_{j k}(x, 0)\right) \sum_{l=1}^{3}\left(-\partial_{l} u_{k}(y)+\delta_{k l} \pi(y)+\left(\tau e_{1}-\omega \times y\right)_{l} u_{k}(y)\right) n_{l}(y)\right. \\
& \quad+\left(E_{4 j}(x-y)-E_{4 j}(x)\right) u_{k}(y) n_{k}(y) \\
& \left.\quad+\sum_{l=1}^{3}\left(\partial y_{l} Z_{j k}(x, y)\left(u_{k} n_{l}\right)(y)+\tau Z_{j k}(x, 0)\left(u_{l} u_{k} n_{l}\right)(y)\right)\right] d o_{y}
\end{aligned}
$$

for $x \in{\overline{B_{S_{1}}}}^{c}, 1 \leq j \leq 3$. The preceding integrals are absolutely convergent. Moreover $\mathfrak{F} \in C^{1}\left({\overline{B_{S_{1}}}}^{c}\right)^{3}$ and equation (1.7) holds. In addition, for any $S \in\left(S_{1}, \infty\right)$, there is a constant $C>0$ which depends on $\tau, \varrho, S_{1}, S, f, u$ and $\pi$, and which is such that

$$
\left|\partial^{\alpha} \mathfrak{F}(x)\right| \leq C(|x| s(x))^{-3 / 2-|\alpha| / 2} \ln (2+|x|) \quad \text { for } x \in{\overline{B_{S}}}^{c}, \alpha \in \mathbb{N}_{0}^{3} \text { with }|\alpha| \leq 1
$$

In the preceding theorem, the coefficients $\beta_{1}, \beta_{2}, \beta_{3}$ and the function $\mathfrak{F}$ are defined in terms of integrals on $\partial \mathfrak{D}$ and $\overline{\mathfrak{D}}^{c}$. The integral over $\partial \mathfrak{D}$ may allow to exploit boundary conditions verified by $u$ or $\pi$. However, this way of introducing $\beta_{1}, \beta_{2}, \beta_{3}$ and $\mathfrak{F}$ requires the additional assumptions imposed on $\mathfrak{D}, u$ and $\pi$ in (3.1). If boundary conditions on $\partial \mathfrak{D}$ 
do not matter, we may drop (3.1) and consider $\left(u\left|{\overline{B_{S_{0}}}}^{c}, \pi\right|{\overline{B_{S_{0}}}}^{c}\right)$ instead of $(u, \pi)$, where $S_{0}$ may be any number from $\left(0, S_{1}\right)$ with $\overline{\mathfrak{D}} \cup \operatorname{supp}(f) \subset B_{S_{0}}$. In view of interior regularity of $u$ and $\pi$, we may then define the coefficients $\beta_{k}$ and the functions $\mathfrak{F}$ in terms of integrals over $\partial B_{S_{0}}$ and $\overline{B_{S_{0}}}$, obtaining an analogous result as the one in Theorem 3.1, but with $B_{S_{0}}$ in the role of $\mathfrak{D}$. Below we will present a variant of this idea which takes account of the additional results in the work at hand (Corollary 3.1).

The principal aim of this article consists in improving Theorem 3.1 in the way specified in

Theorem 3.2 Let $\mathfrak{D}, p, f, S_{1}, u, \pi$ satisfy the assumptions of Theorem 3.1, including (3.1). Let $\beta_{1}, \beta_{2}, \beta_{3}$ and $\mathfrak{F}$ be defined as in Theorem 3.1. Define the function $\mathfrak{G}$ as in (1.10).

Then $\mathfrak{G} \in C^{1}\left({\overline{B_{S_{1}}}}^{c}\right)^{3}$, equation (1.11) holds, and for any $S \in\left(S_{1}, \infty\right)$, there is a constant $C>0$ which depends on $\tau, \varrho, S_{1}, S, f, u$ and $\pi$, and which is such that

$$
\left|\partial^{\alpha} \mathfrak{G}(x)\right| \leq C(|x| s(x))^{-3 / 2-|\alpha| / 2} \ln (2+|x|) \quad \text { for } x \in{\overline{B_{S}}}^{c}, \alpha \in \mathbb{N}_{0}^{3} \text { with }|\alpha| \leq 1
$$

We recall that the asymptotic behaviour of the function $E$ appearing in the leading term in (1.11) is described in Theorem 2.3. As explained above, we may drop the assumptions in (3.1) if we replace $(u, \pi)$ by $\left(u\left|{\overline{B_{S_{0}}}}^{c}, \pi\right|{\overline{B_{S_{0}}}}^{c}\right)$, with some suitably chosen number $S_{0}$. Here are the details.

Corollary 3.1 Take $\mathfrak{D}, p, f, S_{1}, u, \pi$ as in Theorem [3.1, but without requiring (3.1). (This means that $(u, \pi)$ is only assumed to be a Leray solution of (1.1), (1.2).) Put $\widetilde{p}:=\min \{3 / 2, p\}$.

Then $u \in W_{l o c}^{2, \widetilde{p}}\left(\overline{\mathfrak{D}}^{c}\right)^{3}$ and $\pi \in W_{l o c}^{1, \widetilde{p}}\left(\overline{\mathfrak{D}}^{c}\right)$.

Fix some number $S_{0} \in\left(0, S_{1}\right)$ with $\overline{\mathfrak{D}} \cup \operatorname{supp}(f) \subset B_{S_{0}}$, and define $\beta_{1}, \beta_{2}, \beta_{3}$ and $\mathfrak{F}$ as in Theorem [3.1, but with $\mathfrak{D}$ replaced by $B_{S_{0}}$, and $n(x)$ by $S_{0}^{-1} x$, for $x \in \partial B_{S_{0}}$. Moreover, define $\mathfrak{G}$ as in (1.10).

Then all the conclusions of Theorem 3.2 are valid.

\section{Some Fourier transforms.}

In this section we show that $Z_{j 1}(\cdot, 0)=E_{j 1}$. To this end, we prove that the Fourier transforms of these two functions coincide. To begin with, we recall some well known facts about the Fourier transforms of some of the fundamental solutions introduced in Section 2. Other intermediate results in this section may also be well known (Corollary 4.2 for example), but since their proofs are very short, we present them for completeness.

Theorem 4.1 For $\xi \in \mathbb{R}^{3} \backslash\{0\}$, we have $\widehat{N}(\xi)=(2 \pi)^{-3 / 2}|\xi|^{-2}$. If $f \in \mathfrak{S}\left(\mathbb{R}^{3}\right), \xi \in$ $\mathbb{R}^{3} \backslash\{0\}$, and if $F(x):=\int_{\mathbb{R}^{3}} N(x-y) f(y) d y$ for $x \in \mathbb{R}^{3}$, then $\widehat{F}(\xi)=|\xi|^{-2} \widehat{f}(\xi)(\xi \in$ $\left.\mathbb{R}^{3} \backslash\{0\}\right)$.

Moreover $[K(\cdot, t)]^{\wedge}(\xi)=(2 \pi)^{-3 / 2} e^{-t|\xi|^{2}}$ for $\xi \in \mathbb{R}^{3}, t>0$, and $\widehat{\mathfrak{D}^{(\lambda)}}(\xi)=F^{(\lambda)}(\xi)$ for $\xi \in \mathbb{R}^{3}, \lambda \in(0, \infty)$. 
Proof: For the first formula, the reader may consult [50, Proposition 2.1.1] and its proof. The second equation follows from the first by a well known formula for the Fourier transform of a convolution. As a direct reference we mention [56, Lemma V.1.1]. The third equation is well known, and as concerns the forth, we refer to [10, Theorem 2.1].

Corollary 4.1 $\widehat{\mathfrak{D}}(\xi)=(2 \pi)^{-3 / 2}\left(i \tau \xi_{1}+|\xi|^{2}\right)^{-1}$ for $\xi \in \mathbb{R}^{3} \backslash\{0\}$.

Proof: Let $\varphi \in \mathfrak{S}\left(\mathbb{R}^{3}\right)$. For $n \in \mathbb{N}, \xi \in \mathbb{R}^{3}$, we have $\left|F^{(1 / n)}(\xi) \varphi(\xi)\right| \leq \mathfrak{C}|\xi|^{-2}|\varphi(\xi)|$. But $\int_{\mathbb{R}^{3}}|\xi|^{-2}|\varphi(\xi)| d \xi<\infty$, because $\varphi$ is rapidly decreasing. Thus we get from Lebesgue's theorem

$$
\mathfrak{A}:=(2 \pi)^{-3 / 2} \int_{\mathbb{R}^{3}}\left(i \tau \xi_{1}+|\xi|^{2}\right)^{-1} \varphi(\xi) d \xi=\lim _{n \rightarrow \infty} \int_{\mathbb{R}^{3}} F^{(1 / n)}(\xi) \varphi(\xi) d \xi .
$$

Due to the last equation in Theorem 4.1, we may conclude

$$
\mathfrak{A}=\lim _{n \rightarrow \infty} \int_{\mathbb{R}^{3}} \mathfrak{D}^{(1 / n)}(x) \widehat{\varphi}(x) d x .
$$

But $\left|\mathfrak{O}^{(1 / n)}(x) \widehat{\varphi}(x)\right| \leq \mathfrak{C}|x|^{-1}|\widehat{\varphi}(x)|$ for $n \in \mathbb{N}, x \in \mathbb{R}^{3} \backslash\{0\}$, with $\int_{\mathbb{R}^{3}}|x|^{-1}|\widehat{\varphi}(x)| d x<\infty$ because $\varphi$ hence $\widehat{\varphi}$ is rapidly decreasing. Thus equation (4.1) and Lebesgue's theorem yield $\mathfrak{A}=\int_{\mathbb{R}^{3}} \mathfrak{O}(x) \widehat{\varphi}(x) d x$. Since this is true for any $\varphi \in \mathfrak{S}\left(\mathbb{R}^{3}\right)$, the corollary follows.

Corollary 4.2 Let $t \in(0, \infty), j, k \in\{1,2,3\}$. Then

$$
\left[T_{j k}(\cdot, t)\right]^{\wedge}(\xi)=(2 \pi)^{-3 / 2}\left(\delta_{j k}-\xi_{j} \xi_{k}|\xi|^{-2}\right) e^{-t|\xi|^{2}} \quad \text { for } \xi \in \mathbb{R}^{3} \backslash\{0\} .
$$

Proof: We have $K(\cdot, t) \in \mathfrak{S}\left(\mathbb{R}^{3}\right)$ (Lemma 2.5). Therefore by Lemma 2.4, $T_{j k}(x, t)=$ $\delta_{j k} K(x, t)+\int_{\mathbb{R}^{3}} N(x-y) \partial_{j} \partial_{k} K(y, t) d y\left(x \in \mathbb{R}^{3}\right)$. Since $K(\cdot, t)$ belongs to $\mathfrak{S}\left(\mathbb{R}^{3}\right)$ hence $\partial_{j} \partial_{k} K(\cdot, t)$ does, too, Corollary 4.2 follows from Theorem 4.1 .

Corollary 4.3 Let $j \in\{1,2,3\}, t \in(0, \infty)$. Then

$$
\left[\Gamma_{j 1}(\cdot, 0, t)\right]^{\wedge}(\xi)=(2 \pi)^{-3 / 2}\left(\delta_{j 1}-\xi_{j} \xi_{1}|\xi|^{-2}\right) e^{-t\left(i \tau \xi_{1}+|\xi|^{2}\right)} \quad \text { for } \xi \in \mathbb{R}^{3} \backslash\{0\} .
$$

Proof: By Lemma 2.3, we have $\Gamma_{j 1}(x, 0, t)=\left(T\left(x-\tau t e_{1}, t\right) e^{-t \Omega}\right)_{j 1}=T_{j 1}\left(x-\tau t e_{1}, t\right)$, so Corollary 4.3 follows from Corollary 4.2 .

Corollary 4.4 Let $j \in\{1,2,3\}, t \in(0, \infty)$. Then

$$
\left[Z_{j 1}(\cdot, 0)\right]^{\wedge}(\xi)=(2 \pi)^{-3 / 2}\left(\delta_{j 1}-\xi_{j} \xi_{1}|\xi|^{-2}\right)\left(i \tau \xi_{1}+|\xi|^{2}\right)^{-1} \quad \text { for } \xi \in \mathbb{R}^{3} \backslash\{0\} .
$$

Proof: Let $\varphi \in \mathfrak{S}\left(\mathbb{R}^{3}\right)$. With Corollary 2.1, we get

$$
A:=\int_{\mathbb{R}^{3}} \int_{0}^{\infty}\left|\Gamma_{j 1}(x, 0, t) \widehat{\varphi}(t)\right| d t d x \leq \mathfrak{C} \int_{\mathbb{R}^{3}} \int_{0}^{\infty}\left(\left|x-\tau t e_{1}\right|^{2}+t\right)^{-3 / 2}|\widehat{\varphi}(x)| d t d x .
$$


Next we apply Lemma 2.2 to obtain

$$
\begin{aligned}
& A \leq \mathfrak{C}\left(\int_{\mathbb{R}^{3}} \int_{1}^{\infty} t^{-3 / 2}|\widehat{\varphi}(x)| d t d x+\int_{B_{1}} \int_{0}^{1}\left(|x|^{2}+t\right)^{-3 / 2}|\widehat{\varphi}(x)| d t d x\right. \\
& \left.\quad+\int_{B_{1}^{c}} \int_{0}^{1}(|x| s(x)+t)^{-3 / 2}|\widehat{\varphi}(x)| d t d x\right) \\
& \leq \mathfrak{C}\left(\int_{\mathbb{R}^{3}}|\widehat{\varphi}(x)| d x+\int_{B_{1}} \int_{0}^{1}|x|^{-3 / 2} t^{-3 / 4}|\widehat{\varphi}(x)| d t d x+\int_{B_{1}^{c}} \int_{0}^{1}(1+t)^{-3 / 2}|\widehat{\varphi}(x)| d t d x\right) \\
& \leq \mathfrak{C} \int_{\mathbb{R}^{3}}|\widehat{\varphi}(x)|\left(1+|x|^{-3 / 2}\right) d x .
\end{aligned}
$$

Since $\varphi$ hence $\widehat{\varphi}$ belongs to $\mathfrak{S}\left(\mathbb{R}^{3}\right)$, we know that $\int_{\mathbb{R}^{3}}|\widehat{\varphi}(x)|\left(1+|x|^{-3 / 2}\right) d x<\infty$, so $A<\infty$. Therefore we may apply Fubini's theorem, to obtain

$$
\begin{aligned}
& \int_{\mathbb{R}^{3}} Z_{j 1}(x, 0) \widehat{\varphi}(x) d x=\int_{0}^{\infty} \int_{\mathbb{R}^{3}} \Gamma_{j 1}(x, 0, t) \widehat{\varphi}(x) d x d t \\
& =\int_{0}^{\infty} \int_{\mathbb{R}^{3}}(2 \pi)^{-3 / 2}\left(\delta_{j 1}-\xi_{j} \xi_{1}|\xi|^{-2}\right) e^{-t\left(i \tau \xi_{1}+|\xi|^{2}\right)} \varphi(\xi) d \xi d t,
\end{aligned}
$$

where the last equation follows from Corollary 4.3, But

$$
\begin{aligned}
& \int_{0}^{\infty} \int_{\mathbb{R}^{3}}\left|\left(\delta_{j 1}-\xi_{j} \xi_{1}|\xi|^{-2}\right) e^{-t\left(i \tau \xi_{1}+|\xi|^{2}\right)} \varphi(\xi)\right| d \xi d t \leq \mathfrak{C} \int_{0}^{\infty} \int_{\mathbb{R}^{3}} e^{-t|\xi|^{2}}|\varphi(\xi)| d \xi d t \\
& \leq \mathfrak{C} \int_{\mathbb{R}^{3}}|\xi|^{-2}|\varphi(\xi)| d \xi<\infty,
\end{aligned}
$$

with the last relation holding due to the assumption $\varphi \in \mathfrak{S}\left(\mathbb{R}^{3}\right)$. Thus we may use Fubini's theorem, arriving at the equation

$$
\int_{\mathbb{R}^{3}} Z_{j 1}(x, 0) \widehat{\varphi}(x) d x=\int_{\mathbb{R}^{3}}(2 \pi)^{-3 / 2}\left(\delta_{j 1}-\xi_{j} \xi_{1}|\xi|^{-2}\right)\left(i \tau \xi_{1}+|\xi|^{2}\right)^{-1} \varphi(\xi) d \xi .
$$

This proves Corollary 4.4

Theorem 4.2 Let $j, k \in\{1,2,3\}$. Then for $\xi \in \mathbb{R}^{3} \backslash\{0\}$,

$$
\widehat{E}_{j k}(\xi)=(2 \pi)^{-3 / 2}\left(\delta_{j 1}-\xi_{j} \xi_{1}|\xi|^{-2}\right)\left(i \tau \xi_{1}+|\xi|^{2}\right)^{-1} .
$$

Proof: For $x \in \mathbb{R}^{3} \backslash\{0\}$, we find

$$
\begin{aligned}
& \partial_{1} \Phi(x)=(4 \pi \tau)^{-1} \psi^{\prime}\left(\tau\left(|x|-x_{1}\right) / 2\right) \tau\left(x_{1} /|x|-1\right) / 2=(4 \pi \tau|x|)^{-1}\left(e^{-\tau\left(|x|-x_{1}\right) / 2}-1\right) \\
& =\tau^{-1}(\mathfrak{O}(x)-N(x)) .
\end{aligned}
$$

Hence with Corollary 4.1 and Theorem 4.1, for $\xi \in \mathbb{R}^{3} \backslash\{0\}$,

$$
\begin{aligned}
& i \xi_{1} \widehat{\Phi}(\xi)=\widehat{\partial_{1} \Phi}(\xi)=\tau^{-1}(2 \pi)^{-3 / 2}\left(\left(i \tau \xi_{1}+|\xi|^{2}\right)^{-1}-|\xi|^{-2}\right) \\
& =-i(2 \pi)^{-3 / 2} \xi_{1}\left(\left(i \tau \xi_{1}+|\xi|^{2}\right)|\xi|^{2}\right)^{-1} .
\end{aligned}
$$


As a consequence $\widehat{\Phi}(\xi)=-(2 \pi)^{-3 / 2} \xi_{1}\left(\left(i \tau \xi_{1}+|\xi|^{2}\right)|\xi|^{2}\right)^{-1}$, so the theorem follows by the definition of $E_{j k}$.

Theorem 4.2 may be deduced also from the results in [23, Chapter VII]. In fact, it is shown in [23, Section VII.3] that the convolution $\mathfrak{O} * f$, for $f \in C_{0}^{\infty}\left(\mathbb{R}^{3}\right)^{3}$, belongs to $C^{\infty}\left(\mathbb{R}^{3}\right)^{3}$ and is the velocity part of a solution to the Oseen system (1.5) in $\mathbb{R}^{3}$. On the other hand, by [23, Section VII.4], the inverse Fourier transform of the function $(2 \pi)^{-3 / 2}\left(\delta_{j 1}-\right.$ $\left.\xi_{j} \xi_{1}|\xi|^{-2}\right)\left(i \tau \xi_{1}+|\xi|^{2}\right)^{-1} \widehat{f}(\xi)$ also solves (1.5) in $\mathbb{R}^{3}$, and belongs to certain Sobolev spaces. A uniqueness result yields that the two solutions coincide, implying Theorem 4.2 . However, we prefer to carry out a direct proof of this theorem, instead of relying on the rather lengthy theory in [23, Chapter VII], which in fact yields much stronger results, not needed here, than Theorem 4.2 .

Combining Theorem 4.2 and Corollary 4.4, we arrive at the main result of this section.

Corollary $4.5 Z_{j 1}(\cdot, 0)=E_{j 1}$ for $1 \leq j \leq 3$.

\section{Proof of Theorem 3.2 and Corollary 3.1 .}

We first show that in the case $k \in\{2,3\}$, the function $\partial_{j k}^{\alpha} Z(\cdot, 0)$ decays faster for $|x| \rightarrow \infty$ than indicated by Corollary 2.3 .

Theorem 5.1 Let $S \in[2 \tau \pi /|\varrho|, \infty)$. Then $\left|\partial_{x}^{\alpha} Z_{j k}(x, 0)\right| \leq \mathfrak{C}(S)(|x| s(x))^{-3 / 2-|\alpha| / 2}$ for $x \in B_{S+\tau \pi / \varrho \mid}^{c}, \alpha \in \mathbb{N}_{0}^{3}$ with $|\alpha| \leq 1, j \in\{1,2,3\}, k \in\{2,3\}$.

Proof: Take $x, \alpha, j, k$ as in the theorem. We get with Lemma 2.7 that

$$
\partial_{x}^{\alpha} Z_{j k}(x, 0)=\int_{0}^{\infty} \partial_{x}^{\alpha} \Gamma_{j k}(x, 0, t) d t=\int_{0}^{\infty}\left[\partial_{x}^{\alpha} T\left(x-\tau t e_{1}, t\right) \cdot e^{-t \Omega}\right]_{j k} d t
$$

so with Lemma 2.3 in the case $k=2$,

$$
\partial_{x}^{\alpha} Z_{j k}(x, 0)=\int_{0}^{\infty}\left(\partial_{x}^{\alpha} T_{j 2}\left(x-\tau t e_{1}, t\right) \cos (\varrho t)-\partial_{x}^{\alpha} T_{j 3}\left(x-\tau t e_{1}, t\right) \sin (\varrho t)\right) d t,
$$

with a similar formula in the case $k=3$. Let $\sigma: \mathbb{R} \mapsto \mathbb{R}$ be defined by either $\sigma(t):=\cos (\varrho t)$ for $t \in \mathbb{R}$, or by $\sigma(t):=\sin (\varrho t)$ for $t \in \mathbb{R}$. Let $m \in\{1,2,3\}$. Then

$$
\begin{aligned}
& \int_{0}^{\infty} \partial_{x}^{\alpha} T_{j m}\left(x-\tau t e_{1}, t\right) \sigma(t) d t \\
& =\sum_{n=0}^{\infty} \int_{n \pi /|\varrho|}^{(n+1) \pi /|\varrho|}\left(\partial_{x}^{\alpha} T_{j m}\left(x-\tau t e_{1}, t\right)-\partial_{x}^{\alpha} T_{j m}\left(x-\tau(t+\pi /|\varrho|) e_{1}, t+\pi /|\varrho|\right)\right) \sigma(t) d t \\
& =\sum_{n=0}^{\infty} \int_{n \pi /|\varrho|}^{(n+1) \pi /|\varrho|} \int_{0}^{1}\left(-\tau \partial_{x}^{\alpha+e_{1}}+\partial_{x}^{\alpha} \partial_{4}\right) T_{j m}\left(x-\tau(t+\vartheta \pi /|\varrho|) e_{1}, t+\vartheta \pi /|\varrho|\right) \\
& \cdot(-\pi /|\varrho|) \sigma(t) d \vartheta d t .
\end{aligned}
$$


Therefore by Lemma 2.6.

$$
\begin{aligned}
& A:=\left|\int_{0}^{\infty} \partial_{x}^{\alpha} T_{j, m}\left(x-\tau t e_{1}, t\right) \sigma(t) d t\right| \\
& \leq \mathfrak{C} \sum_{n=0}^{\infty} \sum_{m=1}^{2} \int_{n \pi /|\varrho|}^{(n+1) \pi /|\varrho|} \int_{0}^{1}\left(\left|x-\tau(t+\vartheta \pi /|\varrho|) e_{1}\right|^{2}+t+\vartheta \pi /|\varrho|\right)^{-3 / 2-|\alpha| / 2-m / 2} d \vartheta d t \\
& \leq \mathfrak{C} \sum_{m=1}^{2} \int_{0}^{1} \int_{0}^{\infty}\left(\left|x-(\tau \vartheta \pi /|\varrho|) e_{1}-\tau t e_{1}\right|^{2}+t\right)^{-3 / 2-|\alpha| / 2-m / 2} d t d \vartheta .
\end{aligned}
$$

Since $x \in B_{S+\tau \pi /|\varrho|}^{c}$, we have $\left|x-(\tau \vartheta \pi /|\varrho|) e_{1}\right| \geq S$ for $\vartheta \in[0,1]$, so we may apply Theorem 2.1 with $z=0, R_{2}=S, R_{1}=S / 2, y=x-(\tau \vartheta \pi /|\varrho|) e_{1}, \nu=3 / 2+|\alpha| / 2+l / 2$, to obtain

$$
A \leq \mathfrak{C}(S) \sum_{m=1}^{2} \int_{0}^{1}\left[\left|x-(\tau \vartheta \pi /|\varrho|) e_{1}\right| s\left(x-(\tau \vartheta \pi /|\varrho|) e_{1}\right)\right]^{-1-|\alpha| / 2-m / 2} d \vartheta .
$$

But for $\vartheta \in[0,1]$, we have $\left|x-(\tau \vartheta \pi /|\varrho|) e_{1}\right| \geq|x| / 2+S / 2-\tau \vartheta \pi /|\varrho| \geq|x| / 2$, where the last inequality holds because $S \geq 2 \tau \pi /|\varrho|$. Moreover we get from from Lemma 2.1 that $s\left(x-(\tau \vartheta \pi /|\varrho|) e_{1}\right)^{-1} \leq \mathfrak{C} s(x)^{-1}$ for $\vartheta \in[0,1]$. Therefore from (5.2),

$$
A \leq \mathfrak{C}(S) \sum_{m=1}^{2}(|x| s(x))^{-1-|\alpha| / 2-m / 2} \leq \mathfrak{C}(S)(|x| s(x))^{-3 / 2-|\alpha| / 2} .
$$

Theorem 5.1 follows with equation (5.1) and its analogue for $k=3$.

Corollary 5.1 Let $S \in(0, \infty)$. Then $\left|\partial_{x}^{\alpha} Z_{j k}(x, 0)\right| \leq \mathfrak{C}(S)(|x| s(x))^{-3 / 2-|\alpha| / 2}$ for $x \in B_{S}^{c}$ and for $\alpha, j, k$ as in Theorem 5.1.

Proof: Let $x \in B_{S}^{c}$, and take $\alpha, j, k$ as in Theorem [5.1, By Corollary 2.3, we have $\left|\partial_{x}^{\alpha} Z_{j k}(x, 0)\right| \leq \mathfrak{C}(S)(|x| s(x))^{-1-|\alpha| / 2}$.

Suppose that $S \geq 2 \tau \pi /|\varrho|$. Then we distinguish the cases $x \in B_{S+\tau \pi /|\varrho|}^{c}$ and $x \in$ $B_{S+\tau \pi /|\varrho|} \backslash B_{S}$. If $x \in B_{S+\tau \pi /|\varrho|}^{c}$, the looked-for inequality follows from Theorem 5.1. In the second case, we observe that $1 \leq(S+\tau \pi /|\varrho|)|x|^{-1}$, so the inequality claimed in Corollary 5.1 may be deduced from the estimate stated at the beginning of this proof.

Now suppose that $S<2 \tau \pi /|\varrho|$, Then we use that either $x \in B_{3 \tau \pi /|\varrho|}^{c}$ or $x \in B_{3 \tau \pi /|\varrho|} \backslash B_{S}$. If $x \in B_{3 \tau \pi /|\varrho|}^{c}$, the looked-for inequality follows from Theorem 5.1 with $2 \tau \pi /|\varrho|$ in the place of $S$. In the case $x \in B_{3 \tau \pi /|\varrho|} \backslash B_{S}$, we use the relation $1 \leq(3 \tau \pi /|\varrho|)|x|^{-1}$ and again the estimate from the beginning of the proof, once more obtaining an upper bound $\mathfrak{C}(S)(|x| s(x))^{-3 / 2-|\alpha| / 2}$ for $\left|\partial_{x}^{\alpha} Z_{j k}(x, 0)\right|$, as stated in Corollary [5.1,

The proofs of Theorem 3.2 and Corollary 3.1 are now obvious.

Proof of Theorem 3.2, Combine Theorem 3.1, Corollary 4.5 and 5.1,

Proof of Corollary 3.1; From interior regularity of solutions to the Stokes system ([23, Theorem IV.4.1]) and the assumption $f \in L^{p}\left(\mathbb{R}^{3}\right)^{3}$, we may conclude that $u \in W_{l o c}^{2, \widetilde{p}}\left(\overline{\mathfrak{D}}^{c}\right)^{3}$ 
and $\pi \in W_{l o c}^{1, \widetilde{p}}\left(\overline{\mathfrak{D}}^{c}\right)$. More details about this conclusion may be found in the proof of [4, Theorem 5.5]. It follows that $u \mid \partial B_{S_{0}} \in W^{2-1 / \widetilde{p}, \widetilde{p}}\left(\partial B_{S_{0}}\right)^{3}$ and $\pi \mid B_{R} \backslash \overline{B_{S_{0}}} \in L^{\widetilde{p}}\left(B_{R} \backslash \overline{B_{S_{0}}}\right)$ for any $R \in\left(S_{0}, \infty\right)$. Now we may apply Theorem 3.2 with $\mathfrak{D}, f, u, \pi$ replaced by $B_{S_{0}}, f\left|{\overline{B_{S_{0}}}}^{c}, u\right|{\overline{B_{S_{0}}}}^{c}$ and $\pi \mid{\overline{B_{S_{0}}}}^{c}$, respectively. Corollary 3.1 then follows from Theorem 3.2 .

Acknowledgements. The work of Šárka Nečasová acknowledges the support of the GAČR (Czech Science Foundation) project P201-13-00522S in the framework of RVO: 67985840. S.N. would like to thank for fruitful discussions with R. Guenther.

\section{References}

[1] P. Deuring, The single-layer potential associated with the time-dependent Oseen system, in: Proceedings of the 2006 IASME/WSEAS International Conference on Continuum Mechanics. Chalkida, Greeece, May 11-13, 2006, 117-125.

[2] P. Deuring and S. Kračmar, Exterior stationary Navier-Stokes flows in 3D with nonzero velocity at infinity: approximation by flows in bounded domains, Math. Nachr., 269-270 (2004), 86-115.

[3] P. Deuring, S. Kračmar and Š. Nečasová, A representation formula for linearized stationary incompressible viscous flows around rotating and translating bodies, Discrete Contin. Dyn. Syst. Ser. S, 3 (2010), 237-253.

[4] P. Deuring, S. Kračmar and Š. Nečasová, On pointwise decay of linearized stationary incompressible viscous flow around rotating and translating bodies, SIAM J. Math. Anal., 43 (2011), 705-738.

[5] P. Deuring, S. Kračmar and Š. Nečasová, Linearized stationary incompressible flow around rotating and translating bodies: Asymptotic profile of the velocity gradient and decay estimate of the second derivatives of the velocity, J. Differential Equations, 252 (2012), 459-476.

[6] P. Deuring, S. Kračmar and Š. Nečasová, A linearized system describing stationary incompressible viscous flow around rotating and translating bodies: Improved decay estimates of the velocity and its gradient, Discrete Contin. Dyn. Syst., Supplement 2011 (2011), 351-361.

[7] P. Deuring S. Kračmar and Š. Nečasová, Pointwise decay of stationary rotational viscous incompressible flows with nonzero velocity at infinity, J. Differential Equations, 255 (2013), 1576-1606.

[8] P. Deuring, S. Kračmar and Š. Nečasová, Linearized stationary incompressible flow around rotating and translating bodies - Leray solutions, Discrete Contin. Dyn. Syst. Ser. S, 7 (2014), 967-979.

[9] P. Deuring, S. Kračmar and Š. Nečasová, Leading terms of velocity and its gradient of the stationary rotational viscous incompressible flows with nonzero velocity at infinity, submitted. 
[10] P. Deuring and W. Varnhorn, On Oseen resolvent estimates, Differ. Integr. Equat., 23 (2010), 1139-1149.

[11] S. D. Eidelman, On fundamental solutions of parabolic systems II, Mat. Zbornik, 53 (1961), 73-136 (Russian).

[12] R. Farwig, An $L^{q}$-analysis of viscous fluid flow past a rotating obstacle, Tôhoku Math. J., 58 (2006), 129-147.

[13] R. Farwig, Estimates of lower order derivatives of viscous fluid flow past a rotating obstacle, Banach Center Publications, 70 (2005), 73-84.

[14] R. Farwig, G. P. Galdi and M. Kyed, Asymptotic structure of a Leray solution to the Navier-Stokes flow around a rotating body, Pacific J. Math., 253 (2011), 367-382.

[15] R. Farwig and T. Hishida, Stationary Navier-Stokes flow around a rotating obstacle, Funkcialaj Ekvacioj, 50 (2007), 371-403.

[16] R. Farwig and T. Hishida, Asymptotic profiles of steady Stokes and Navier-Stokes flows around a rotating obstacle, Ann. Univ. Ferrara, Sez. VII, 55 (2009), 263-277.

[17] R. Farwig and T. Hishida, Asymptotic profile of steady Stokes flow around a rotating obstacle, Manuscripta Math., 136 (2011), 315-338.

[18] R. Farwig and T. Hishida, Leading term at infinity of steady Navier-Stokes flow around a rotating obstacle, Math. Nachr., 284 (2011), 2065-2077.

[19] R. Farwig, T. Hishida and D. Müller, $L^{q}$-theory of a singular "winding" integral operator arising from fluid dynamics, Pacific J. Math., 215 (2004), 297-312.

[20] R. Farwig, M. Krbec and Š. Nečasová, A weighted $L^{q}$ approach to Stokes flow around a rotating body, Ann. Univ. Ferrara, Sez. VII, 54 (2008), 61-84.

[21] R. Farwig, M. Krbec and Š. Nečasová, A weighted $L^{q}$-approach to Oseen flow around a rotating body, Math. Meth. Appl. Sci., 31 (2008), 551-574.

[22] R. Farwig and J. Neustupa, On the spectrum of a Stokes-type operator arising from flow around a rotating body, Manuscripta Math., 122 (2007), 419-437.

[23] G. P. Galdi, An Introduction to the Mathematical Theory of the Navier-Stokes Equations. Vol. I. Linearised Steady Problems, Springer Tracts in Natural Philosophy, 38, Springer, New York e.a., 1998.

[24] G. P. Galdi, On the motion of a rigid body in a viscous liquid: A mathematical analysis with applications, in Handbook of Mathematical Fluid Dynamics. Vol. I (eds. S. Friedlander and D. Serre), North-Holland, Amsterdam, 2002, 653-791.

[25] G.P. Galdi, Steady flow of a Navier-Stokes fluid around a rotating obstacle, J. Elast., 71 (2003), 1-31.

[26] G. P. Galdi, An Introduction to the Mathematical Theory of the Navier-Stokes Equations. Steady-State Problems, 2nd edition, Springer, New York e.a., 2011. 
[27] G. P. Galdi and M. Kyed, Steady-state Navier-Stokes flows past a rotating body: Leray solutions are physically reasonable, Arch. Rat. Mech. Anal., 200 (2011), 21-58.

[28] G. P. Galdi and M. Kyed, Asymptotic behavior of a Leray solution around a rotating obstacle, Progress in Nonlinear Differential Equations and Their Applications, 60 (2011), 251-266.

[29] G. P. Galdi and M. Kyed, A simple proof of $L^{q}$-estimates for the steady-state Oseen and Stokes equations in a rotating frame. Part I: Strong solutions, Proc. Am. Math. Soc., 141 (2013), 573-583.

[30] G. P. Galdi and M. Kyed, A simple proof of $L^{q}$-estimates for the steady-state Oseen and Stokes equations in a rotating frame. Part II: Weak solutions, Proc. Am. Math. Soc., 141 (2013), 1313-1322.

[31] G. P. Galdi and A. L. Silvestre, Strong solutions to the Navier-Stokes equations around a rotating obstacle, Arch. Rat. Mech. Anal., 176 (2005), 331-350.

[32] G. P. Galdi and A. L. Silvestre, The steady motion of a Navier-Stokes liquid around a rigid body, Arch. Rat. Mech. Anal., 184 (2007), 371-400.

[33] G. P. Galdi and A. L. Silvestre, Further results on steady-state flow of a NavierStokes liquid around a rigid body. Existence of the wake, RIMS Kôkyûroku Bessatsu, B1 (2008), 108-127.

[34] M. Geissert, H. Heck and M. Hieber, $L^{p}$ theory of the Navier-Stokes flow in the exterior of a moving or rotating obstacle, J. Reine Angew. Math., 596 (2006), 45-62.

[35] T. Hishida, An existence theorem for the Navier-Stokes flow in the exterior of a rotating obstacle, Arch. Rat. Mech. Anal., 150 (1999), 307-348.

[36] T. Hishida, The Stokes operator with rotating effect in exterior domains, Analysis, 19 (1999), 51-67.

[37] T. Hishida, $L^{q}$ estimates of weak solutions to the stationary Stokes equations around a rotating body, J. Math. Soc. Japan, 58 (2006), 744-767.

[38] T. Hishida and Y. Shibata, Decay estimates of the Stokes flow around a rotating obstacle, RIMS Kôkyûroku Bessatsu, B1 (2007), 167-186.

[39] T. Hishida and Y. Shibata, $L_{p}-L_{q}$ estimate of the Stokes operator and Navier-Stokes flows in the exterior of a rotating obstacle, Arch. Rat. Mech. Anal., 193 (2009), $339-421$.

[40] S. Kračmar, M. Krbec, Š. Nečasová, P. Penel and K. Schumacher, On the $L^{q}$-approach with generalized anisotropic weights of the weak solution of the Oseen flow around a rotating body, Nonlinear Analysis, 71 (2009), e2940-e2957.

[41] S. Kračmar, Š. Nečasová and P. Penel, Estimates of weak solutions in anisotropically weighted Sobolev spaces to the stationary rotating Oseen equations, IASME Transactions, 2 (2005), 854-861. 
[42] S. Kračmar, Š. Nečasová and P. Penel, Anisotropic $L^{2}$ estimates of weak solutions to the stationary Oseen type equations in $\mathbb{R}^{3}$ for a rotating body, RIMS Kôkyûroku Bessatsu, B1 (2007), 219-235.

[43] S. Kračmar, Š. Nečasová and P. Penel, Anisotropic $L^{2}$ estimates of weak solutions to the stationary Oseen type equations in $3 D$ - exterior domain for a rotating body, J. Math. Soc. Japan, 62 (2010), 239-268.

[44] S. Kračmar, A. Novotný and M. Pokorný, Estimates of Oseen kernels in weighted $L^{p}$ spaces, J. Math. Soc. Japan, 53 (2001), 59-111.

[45] S. Kračmar and P. Penel, Variational properties of a generic model equation in exterior 3D domains, Funkcialaj Ekvacioj, 47 (2004), 499-523.

[46] S. Kračmar and P. Penel, New regularity results for a generic model equation in exterior 3D domains, Banach Center Publications Warsaw, 70 (2005), 139-155.

[47] M. Kyed, Asymptotic profile of a linearized flow past a rotating body, Quart. Appl. Math., 71 (2013), 489-500.

[48] M. Kyed, On a mapping property of the Oseen operator with rotation, Discrete Contin. Dynam. Syst. Ser. S, 6 (2013), 1315-1322.

[49] M. Kyed, On the asymptotic structure of a Navier-Stokes flow past a rotating body, J. Math. Soc. Japan, 66 (2014), 1-16.

[50] U. Neri, Singular Integrals, Lecture Notes in Mathematics, 200, Springer, Berlin e.a., 1971.

[51] Š. Nečasová, Asymptotic properties of the steady fall of a body in viscous fluids, Math. Meth. Appl. Sci., 27 (2004), 1969-1995.

[52] Š. Nečasová, On the problem of the Stokes flow and Oseen flow in $\mathbb{R}^{3}$ with Coriolis force arising from fluid dynamics, IASME Transaction, 2 (2005), 1262-1270.

[53] Š. Nečasová and K. Schumacher, Strong solution to the Stokes equations of a flow around a rotating body in weighted $L^{q}$ spaces, Math. Nachr., 284 (2011), 1701-1714.

[54] V. A. Solonnikov, A priori estimates for second order parabolic equations, Trudy Mat. Inst. Steklov., 70 (1964), 133-212 (Russian); English translation: AMS Translations, 65 (1967), 51-137.

[55] V. A. Solonnikov, Estimates of the solutions of a nonstationary linearized system of Navier-Stokes equations, Trudy Mat. Inst. Steklov., 70 (1964), 213-317 (Russian); English translation : AMS Translations, 75 (1968), 1-116.

[56] E. M. Stein, Singular integrals and differentiability of functions, Princeton University Press, Princeton N.J., 1970.

[57] E. A. Thomann and R. B. Guenther, The fundamental solution of the linearized Navier-Stokes equations for spinning bodies in three spatial dimensions - time dependent case, J. Math. Fluid Mech., 8 (2006), 77-98. 